\title{
Impacto da Creche para a Interação Mãe-Criança e para o Desenvolvimento Infantil*
}

\author{
Scheila Machado da Silveira Becker ${ }^{1, * *} \&$ Cesar Augusto Piccinini² \\ ${ }^{1}$ Faculdades Integradas de Taquara, Taquara, RS, Brasil \\ ${ }^{2}$ Universidade Federal do Rio Grande do Sul, Porto Alegre, RS, Brasil
}

\begin{abstract}
RESUMO - Buscou-se investigar o impacto da creche na interação mãe-criança e no desenvolvimento infantil nos dois primeiros anos de vida da criança. Participaram 44 crianças $(M=6,4$ meses; $D P=1,6)$ e suas mães $(M=34,1$ anos; $\mathrm{DP}=5,2)$, divididas em dois grupos: Grupo Creche (21 crianças que frequentavam a creche) e Grupo Não Creche (23 que não frequentavam). Avaliou-se o desenvolvimento aos 6,12 e 18 meses da criança e a interação mãe-criança aos 12 e 18 meses. Os resultados revelaram que a frequência à creche não interferiu na qualidade da interação mãe-criança e não diferenciou os grupos quanto ao desenvolvimento infantil (cognitivo, linguagem, socioemocional), indicando que famílias de classes média e alta e creches com qualidade suficiente podem se igualar em seus eventuais benefícios.
\end{abstract}

PALAVRAS-CHAVE: creche, interação mãe-criança, desenvolvimento infantil

\section{Effects of Childcare Centers on Mother-Child Interaction and Child Development}

\begin{abstract}
The present study investigated the impact of childcare centers on mother-child interaction and on child development in the first two years of a child's life. The study was comprised of 44 children $(\mathrm{M}=6.4 \mathrm{months}, \mathrm{SD}=1.6)$ and their mothers $(\mathrm{M}=34$.1years, $\mathrm{SD}=5.2)$ divided into two groups: Childcare Center Group $(\mathrm{N}=21)$ and Non-Childcare Center Group ( $\mathrm{N}=23)$. The infant development was evaluated at 6,12 and 18 months and mother-child interaction was assessed at the 12th and 18th months. The results revealed that childcare centers did not affect the quality of mother-child interaction and did not set apart the groups in relation to child development (cognitive, language, socioemotional), indicating that middle and upper class families and qualified childcare centers can be equally beneficial.
\end{abstract}

KEYWORDS: childcare center, mother-child interaction, infant development

A creche passou a ser um importante contexto de desenvolvimento para muitas crianças, frente às mudanças econômicas e culturais da sociedade. No entanto, devido à indiscutível importância do contexto familiar para o desenvolvimento infantil, muitas vezes a entrada da criança na creche, especialmente durante o primeiro ano de vida, tem gerado controvérsias, sobretudo porque envolve a transição do ambiente familiar, em que os cuidados tendem a ser íntimos e exclusivos, para um ambiente de cuidado coletivo.

Apesar das dúvidas ainda geradas por esse tema, o aumento da demanda por atendimento de crianças pequenas e a expansão destes serviços no país é uma realidade (Instituto Nacional de Estudos e Pesquisas Educacionais
Anísio Teixeira [Inep], 2012) e a discussão acerca dos possíveis benefícios e prejuízos para o desenvolvimento infantil ganhou força tanto na sociedade como no meio acadêmico. Em função disso, diversos pesquisadores no Brasil (Lordelo, 1997; Lordelo, Chalhub, Guirra, \& Carvalho, 2007) e no exterior (Belsky et al., 2007; Belsky, 2009a; Eunice Kennedy Shriver National Institute of Child Health and Human Development [NICHD], 2003; Sylva et al., 2011) têm se envolvido em estudos que buscam identificar quais seriam os efeitos de cuidados não parentais nos anos iniciais.

A revisão da literatura realizada por Burger (2010), em que foram analisados os achados de estudos europeus,

\footnotetext{
* Apoio: CNPq e Capes

** E-mail: scheilamachado@gmail.com

- Submetido: 11/08/2015; Revisado: 16/05/2016; Aceito: 10/02/2017.
} 
norte-americanos e asiáticos de grande escala, revelou que a educação infantil poderia oferecer às crianças provenientes de contextos de baixa renda e baixa escolaridade um começo mais favorável na escola. Para o autor, isso indica a possibilidade da educação infantil promover $o$ desenvolvimento cognitivo infantil por meio da promoção de experiências sociais e cognitivas complementares ao ambiente familiar.

Os benefícios relacionados à educação infantil também foram revelados pela metanálise realizada por Camilli, Vargas, Ryan e Barnett (2010), que analisou 123 estudos conduzidos ao longo de cinco décadas. Com relação ao desenvolvimento cognitivo, as análises ofereceram evidências de que os programas pré-escolares forneceriam benefícios reais e duradouros para as crianças. Em particular, destacaram os resultados positivos relacionados às habilidades sociais das crianças e ao seu progresso escolar.

Contudo, alguns estudos, por reconhecer que o impacto da creche está relacionado à qualidade do atendimento oferecido, passaram a incluir essa variável em suas análises. De modo geral, tem-se enfatizado que a alta qualidade do cuidado estaria associada a benefícios para o desenvolvimento cognitivo, social e da linguagem infantil (Albers, Riksen-Walraven, \& Weerth, 2010; Burchinal, Vandergrift, Pianta, \& Mashburn, 2010; NICHD, 2002; Ruzek, Burchinal, Farkas, \& Duncan, 2014). Além disso, pesquisas sugerem que os efeitos positivos de cuidados alternativos de alta qualidade são maiores para crianças que estão em ambiente familiar de baixos níveis de renda e escolaridade (Geoffroy et al., 2007; Hungerford \& Cox, 2006; Melhuish, 2004). Estudos brasileiros também indicam que não haveria efeitos negativos para o desenvolvimento infantil em função da separação da mãe, caso a creche seja de boa qualidade, podendo resultar em benefícios reais, especialmente àquelas crianças provenientes de contextos de extrema carência (Kude, 2004; Lordelo et al., 2007).

Entre os estudos nacionais sobre o efeito da experiência de creche para o desenvolvimento infantil, destaca-se o estudo realizado por Lordelo et al. (2007), o qual verificou que o desenvolvimento cognitivo, avaliado por meio de escalas, esteve associado à renda familiar e, parcialmente, à escolaridade materna. Já a frequência à creche não produziu resultados significativos no desempenho cognitivo quando comparada às crianças que não frequentavam a creche. Outro estudo nacional buscou avaliar o impacto da creche e pré-escola na longevidade escolar, ou seja, na conclusão do Ensino Fundamental e do Ensino Básico (Damiani, Dumith, Horta, \& Gigante, 2011). As análises estatísticas indicaram que frequentar a creche não esteva associado à longevidade escolar, mas frequentar a pré-escola apresentou associação significativa. Mesmo controlando o efeito de algumas variáveis, tais como renda familiar, escolaridade materna, sexo e grupo étnico da criança, os sujeitos que frequentaram a pré-escola apresentaram 1,88 vezes mais chance de longevidade escolar no Fundamental e 1,66\% no
Básico do que aqueles que não frequentaram a Educação Infantil. Uma possível explicação para a falta de efeito da creche na longevidade escolar está no fato de o estudo ter iniciado na década de 80 , antes de a creche fazer parte do sistema de ensino (Brasil, 1996) e, portanto, não focar suas atividades nas questões relacionadas ao ensino, como já ocorria com a pré-escola, mas sim prestar um serviço ligado à assistência social.

Outro estudo nacional, encomendado pela Secretaria de Assuntos Estratégicos - (SAE; Barros, Carvalho, Franco, Mendonça, \& Rosalém, 2011), buscou avaliar a relação entre custo, qualidade do atendimento e desenvolvimento infantil. $\mathrm{O}$ estudo avaliou a qualidade de 100 creches municipais e conveniadas da cidade do Rio de Janeiro. Também foi avaliado o desenvolvimento em cerca de 1000 crianças por meio de um instrumento que fornecia informações sobre a idade de desenvolvimento (em meses), em três dimensões (mental, física e social), além de uma medida global de desenvolvimento. Verificou-se que as crianças que frequentaram creches de alta qualidade apresentavam 1,2 meses de idade a mais na avaliação de seu desenvolvimento do que as crianças que frequentam creche de baixa qualidade. Tal diferença foi considerada pelos pesquisadores como moderada, contudo, pode-se pensar que essa diferença é muito pequena e pode refletir a baixa qualidade das creches, até mesmo daquelas que foram consideradas como de alta qualidade pelo estudo, vide seu valor mediano na avaliação. Ainda em relação ao estudo de Barros et al. (2011), os autores verificaram que as crianças que frequentaram creches consideradas de alta qualidade obtiveram escores maiores em seu desenvolvimento mental (1,8 meses a mais) e social (2,3 meses a mais), embora não tenha sido observada diferença em seu desenvolvimento físico.

Além dos possíveis efeitos que a experiência de creche pode trazer para o desenvolvimento infantil, alguns estudos têm investigado se a experiência de creche afetaria a qualidade da interação mãe-criança, frente à hipótese de que, quanto mais horas mães e crianças passariam separadas, mais difícil seria para a mãe aprender sobre os sinais da criança e seu ritmo, afetando assim a qualidade da interação mãe-criança e o desenvolvimento infantil. Nesse sentido, Booth, Clarke-Stewart, Vandell, McCartney e Owen (2002) compararam 143 famílias norte-americanas nas quais as crianças, com seis meses, recebiam mais de 30 horas de cuidado alternativo semanal e 183 famílias nas quais elas recebiam cuidados maternos exclusivos. Os resultados revelaram que as crianças que estavam em casa em tempo integral recebiam mais cuidado instrumental e havia mais interação social com suas mães do que crianças em cuidado alternativo. No entanto, nos finais de semana, os grupos não diferiram na quantidade de cuidado instrumental, e as mães de crianças em cuidado alternativo passaram mais tempo em interação social com os filhos do que as mães do outro grupo. Assim, as mães de crianças que recebiam cuidados alternativos pareciam buscar compensar o tempo longe 
dos filhos, aumentando a interação social com eles quando estavam em casa. Apesar das diferenças significativas entre os grupos no uso do tempo materno, verificou-se que os grupos não diferiram significativamente na qualidade das interações das mães com suas crianças.

Também interessado em investigar o impacto dos cuidados alternativos na qualidade da interação mãecriança, o estudo do NICHD (2003) avaliou a qualidade e a quantidade de cuidado alternativo recebido nos primeiros três anos de vida da criança e a qualidade da interação mãe-criança aos 3, 4 e 5 anos e quando a criança estava na primeira série. Verificou-se que a qualidade do cuidado alternativo nos primeiros anos apresentou associação positiva com a sensibilidade materna quando as crianças tinham experimentado relativamente poucas horas de cuidados alternativos. Segundo os autores, características de um cuidado de alta qualidade, como, por exemplo, parcerias mais estreitas entre os educadores e os pais, envolvendo o compartilhamento de informações sobre a criança, podem favorecer o cuidado mais positivo por parte dos educadores e das próprias mães. Além disso, os educadores poderiam ser potencialmente bons modelos de cuidado positivo para as mães, o que pode ter contribuído para elevar a qualidade da interação mãe-criança.

Diante do exposto, o presente estudo teve como objetivos investigar a qualidade da interação mãe-criança e o desenvolvimento infantil (cognitivo, linguagem, socioemocional e motor) em crianças que frequentaram ou não a creche nos dois primeiros anos de vida. Considerando a literatura (NICHD, 2003), uma das hipóteses era de que a frequência à creche traria benefícios para a interação mãe-criança. Além disso, esperava-se que crianças que frequentavam a creche teriam escores mais elevados de desenvolvimento infantil, especialmente nas escalas de desenvolvimento cognitivo e da linguagem (NICHD, 2006a, 2006b; Ruzek et al., 2014; Sylva et al., 2011).

\section{MÉTODO}

\section{Participantes}

Participaram 44 crianças que, no início do estudo, tinham em média 6 meses de idade $(M=6,4 ; D P=1,6)$ e suas mães $(M=34,1$ anos; $\mathrm{DP}=5,2)$. Desse total, 21 crianças, que compõem o Grupo Creche, ingressaram na creche entre uma semana antes ou após o início do estudo; outras 23 crianças, que compõem o Grupo Não Creche, permaneceram sendo cuidadas em casa, seja pelos pais, avós ou babá. Nenhuma criança da amostra nem suas mães possuíam problemas graves de saúde. A Tabela 1 apresenta as características sociodemográficas dos Grupos Creche e Não Creche na fase dos 6 meses. Utilizou-se o Teste t e o Qui-quadrado $\left(\chi^{2}\right)$ para comparar as diversas características sociodemográficas dos dois grupos, entretanto não foram reveladas diferenças significativas.

As crianças do Grupo Creche ingressaram no berçário de duas creches públicas federais:14 crianças ingressaram na Creche 1 e a média de permanência delas na instituição era de 42,5 horas semanais (aproximadamente 8,5 horas diárias); as outras sete crianças ingressaram na Creche 2 e a média de permanência na instituição foi de 38 horas semanais (aproximadamente 7,6 horas diárias). A qualidade do atendimento dessas creches foi avaliada por meio da escala ITERS-R (Infant/ Toddler Environment Rating Scale - Revised Edition; Harms, Cryer, \& Clifford, 2003). De modo geral, verificou-se que as creches não se diferenciavam substancialmente quanto ao nível de qualidade oferecido às crianças, apresentando nível suficiente de qualidade, conforme classificação sugerida pelos autores (ver também Becker, 2014). Isto indica a existência de condições mínimas de atendimento em ambas as creches, embora não tenham atingido, na avaliação da ITERS, um nível de alta qualidade, que indicaria o oferecimento de condições para a promoção de desenvolvimento infantil (Bairrão, Leal, Lima, \& Morgado, 1997).

Todos os participantes integravam um estudo maior, intitulado Impacto da creche no desenvolvimento socioemocional e cognitivo infantil: Estudo longitudinal do sexto mês de vida do bebê ao final dos anos préescolares, 2010-2016(CRESCI; Piccinini et al., 2010), o qual foi aprovado pelo Comitê de Ética e Pesquisa da Universidade Federal do Rio Grande do Sul - UFRGS (processo $n^{\circ}$ 2010070) e pelo Comitê de Ética e Pesquisa do Hospital de Clínicas de Porto Alegre (processo $\mathrm{n}^{\circ}$ 100553). Esse projeto tem como objetivo analisar o impacto da creche no desenvolvimento socioemocional e cognitivo de crianças, desde seu sexto mês até o final dos anos pré-escolares. Mais especificamente, busca comparar, ao longo desse período, o desenvolvimento de crianças que frequentaram ou não a creche e relacioná-lo com a qualidade dos ambientes institucional e familiar. O projeto iniciou em 2010, acompanhando 77 famílias, entre essas 29 de bebês que frequentavam a creche (Grupo Creche) e 48 de bebês que eram cuidados prioritariamente pela mãe ou por outras cuidadoras, tais como babás (Grupo Não Creche). As famílias cujos bebês ingressaram na creche foram recrutadas para o estudo em duas creches públicas federais e as demais famílias foram chamadas através de e-mail disponibilizado pelo setor de recursos humanos das instituições mantenedoras das duas creches envolvidas no estudo, bem como pela mídia ou por indicações. Além das famílias, o estudo também contou com a participação de 18 educadoras das duas creches. $\mathrm{O}$ projeto envolveu seis fases 
Tabela 1

Características sociodemográficas dos participantes e famílias na fase dos 6 meses nos grupos Creche e Não Creche

\begin{tabular}{|c|c|c|c|c|c|c|}
\hline & \multicolumn{2}{|c|}{ Grupo Creche } & \multicolumn{2}{|c|}{ Grupo Não Creche } & & \multirow{2}{*}{$\mathbf{P}$} \\
\hline & $\%(F)$ & M (DP) & $\%(F)$ & M (DP) & & \\
\hline $\begin{array}{l}\text { Idade da criança } \\
\text { no inicio do estudo }\end{array}$ & & $5,9(1,3)$ & & $6,8(1,8)$ & $\mathrm{t}=-1,7(g l=40,5)$ & 0,09 \\
\hline \multicolumn{7}{|l|}{ Sexo da criança } \\
\hline Menino & $43 \%$ ( 9 ) & & $48 \%(11)$ & & \multirow{2}{*}{$\chi^{2}=0,11(g l=1)$} & \multirow{2}{*}{0,74} \\
\hline Menina & $57 \%(12)$ & & $52 \%(12)$ & & & \\
\hline Idade Mãe & & $34,7(4,4)$ & & $33,6(5,8)$ & $\mathrm{t}=0,7(g l=42)$ & 0,50 \\
\hline Escolaridade Mãe (anos) & & $17,2(2,2)$ & & $18,0(3,6)$ & $\mathrm{t}=-0,9(g l=42)$ & 0,34 \\
\hline Médio Incompleto & - & & $4 \%(1)$ & & & \\
\hline Médio Completo & - & & $4 \%(1)$ & & & \\
\hline Superior Incompleto & $19 \%(4)$ & & $4 \%(1)$ & & $\chi^{2}=4,01(g l=4)$ & 0,30 \\
\hline Superior Completo & $33 \%(7)$ & & $40 \%$ ( 9$)$ & & & \\
\hline Pós Graduação & $48 \%(10)$ & & $48 \%(11)$ & & & \\
\hline \multicolumn{7}{|l|}{ Estado Civil } \\
\hline Casada & $91 \%(19)$ & & $96 \%(22)$ & & \multirow{2}{*}{$\chi^{2}=0,67(g l=1)$} & \multirow{2}{*}{0,89} \\
\hline Solteira & $9 \%(2)$ & & $4 \%(1)$ & & & \\
\hline Número de filhos & & $1,1(0,3)$ & & $1,3(0,5)$ & $\mathrm{t}=-1,2(\mathrm{gl} l=40,7)$ & 0,20 \\
\hline Renda Familiar (R\$) & & $6671(1999)$ & & 9802 (8099) & $\mathrm{t}=-1,8(g l=24,9)$ & 0,09 \\
\hline
\end{tabular}

Grupo Creche: n=21; Grupo Não Creche: n=23. 'Salário Mínimo (SM) em $2011=\mathrm{R} \$ 545$.

de coleta de dados: $6^{\circ}, 12^{\circ}, 18^{\circ}, 24^{\circ}, 36^{\circ}$ e $48^{\circ}$ mês de vida da criança. Ao longo desse período, o desenvolvimento das crianças está sendo avaliado e são aplicadas entrevistas e questionários com as mães e os pais, com o objetivo de avaliar sua percepção sobre o desenvolvimento da criança, bem como de verificar a qualidade do ambiente familiar. Cabe ressaltar que foram também realizadas filmagens da interação livre mãe-bebê e pai-bebê (no $6^{\circ}, 12^{\circ}$ e $18^{\circ}$ mês). Por sua vez, a qualidade das creches também foi avaliada e as educadoras preencheram questionários sobre sua percepção a respeito da adaptação à creche e desenvolvimento da criança (no $6^{\circ}, 12^{\circ}$ e $18^{\circ}$ mês).

Para fins do presente estudo, foram escolhidas do projeto CRESCI todas as crianças que permaneceram sob a mesma forma de cuidado (Creche ou Não Creche) durante as três primeiras fases do projeto $\left(6^{\circ}, 12^{\circ}\right.$ e $18^{\circ}$ mês de vida) e que tinham todos os dados completos. Quanto ao Grupo Não Creche, também foram considerados na seleção dos participantes a idade e o sexo das crianças, pois se buscou compor um grupo semelhante ao Grupo Creche.

\section{Delineamento e Procedimento}

Trata-se de um estudo longitudinal que acompanhou as mesmas crianças em três fases de coleta de dados: $6^{\circ}$, $12^{\circ}$ e $18^{\circ}$ mês de vida da criança. Por volta dos 6 meses de vida da criança, as famílias foram convidadas a participar do Projeto CRESCI. Aquelas que estavam ingressando na creche foram convidadas no momento da matrícula da criança, já as que não iriam para a creche foram convidadas através de e-mail, bem como pela mídia ou por indicações. As famílias que aceitaram participar e atendiam aos critérios do estudo preencheram a Ficha de Dados Demográficos da Família. No mesmo encontro, avaliou-se o desenvolvimento do bebê através das Escalas Bayley III. Poucos dias depois, realizou-se a Observação da Interação Mãe-Bebê - $6^{\circ}$ mês no Laboratório de Observação de Processos Interativos (LOPI) do Instituto de Psicologia da UFRGS. Nas fases dos 12 e dos 18 meses de idade, foram repetidos os mesmos procedimentos de coleta de dados.

\section{Instrumentos}

A seguir, destacam-se apenas os instrumentos cujos dados serão utilizados no presente estudo. Detalhes dos demais instrumentos usados em cada fase do Projeto CRESCI encontram-se em Piccinini et al. (2010).

Ficha de dados demográficos da família (Núcleo de Infância e Família [NUDIF]/CRESCI, 2011a). Visa obter alguns dados demográficos, tais como a idade da mãe e do companheiro, escolaridade, profissão, estado civil, existência de outros filhos, religião, tempo de trabalho etc. 
Escalas Bayley III (Bayley, 2006). Foi utilizada para avaliação do desenvolvimento das habilidades cognitivas, motoras, de linguagem e socioemocional dos bebês. Tratase de um instrumento que pode ser usado entre o $15^{\circ}$ dia ao $42^{\circ}$ mês de idade do bebê e sua aplicação foi realizada por uma psicóloga devidamente habilitada. Os resultados das escalas são expressos em escores normalizados, que variam entre 40 e 160 pontos (média 100 e desvio padrão 15). Embora a variação nesses escores possa servir como critério de classificação do nível de desenvolvimento, no presente estudo foram utilizados os escores brutos como indicadores do desenvolvimento da criança.

Observação da Interação Mãe-Bebê - $1^{\circ}$ mês e $1^{\circ}$ mês (NUDIF/CRESCI, 2011b). Trata-se de sessão de observação filmada de 30 minutos de interação livre mãe-criança, realizada no na sala do LOPI. A sala possui aproximadamente $20 \mathrm{~m}^{2}$, duas câmeras de vídeo ocultas por cortinas, quatro cadeiras, tatame de EVA centralizado com aproximadamente $4 \mathrm{~m}^{2}$, revistas e brinquedos apropriados para a faixa etária. Ao iniciar a sessão, solicitou-se às mães que interagissem livremente com o filho, como faziam quando estavam juntos.

Para fins de análise da qualidade da interação mãecriança aos 12 e 18 meses do bebê, foi utilizado o Maternal Behavior Q-sort (MBQS; Pederson, Moran, \& Bento, 2013), instrumento composto por 90 itens que descrevem uma variedade de comportamentos interativos materno. Os itens são organizados em nove domínios: (1) Estar ciente dos sinais do filho - reflete o quanto a mãe está consciente do filho e de suas necessidades. Uma mãe com alto escore neste domínio está constantemente alerta aos sinais da criança, independentemente de tarefas ou demandas concorrentes. Nos casos de baixo escore, contudo, ela demonstra lapsos de consciência das necessidades da criança e dos seus sinais e é indiferente aos sinais que o filho emite; (2) Eficácia de resposta - avalia a eficácia da resposta da mãe aos sinais do filho. Uma mãe com escore elevado responde consistentemente e com eficácia a todos os sinais, tanto os óbvios quanto os sutis, de tal modo que satisfaça a criança. A mãe com baixo escore neste domínio ou não responde aos sinais do filho, ou quando o faz, as intervenções não são apropriadas; (3) Afeto positivo - avalia a forma como o afeto/ emoção da mãe influencia sua interação com o filho. A mãe com alto escore neste domínio expressa claramente seus sentimentos positivos em relação ao bebê, enquanto uma mãe com baixo escore é caracterizada pela falta de afeto; (4) Rejeição - avalia o quanto a mãe aceita o filho e seus comportamentos. A mãe com alto escore neste domínio rejeita ativamente a criança. Ela é rápida para criticar e punir seu filho ou suas ações. A mãe com baixo escore não expressa irritação e frustração para o filho; (5) Sincronia - avalia o grau em que a mãe e o filho estão envolvidos em interação recíproca, além da capacidade da mãe para uma transição suave entre as atividades e interações com a criança. A mãe com alto escore neste domínio é capaz de adaptar seu comportamento para atender interesses do filho e as interações não são abruptamente iniciadas nem encerradas. Já a mãe com baixo escore não se envolve em interação harmoniosa com o filho; (6) Controle e interferência - avalia a forma como a mãe orienta o comportamento do filho, considerando o quanto a mãe interfere nos comportamentos autônomos da criança durante a interação. Uma mãe com alto escore neste domínio mostra pouco respeito por seu filho e age de acordo com seu próprio interesse. Já a mãe com baixo escore parece apoiar, ao invés de controlar, as interações e interfere o mínimo possível. Essa mãe permite que a criança participe da interação de acordo com seus próprios desejos; (7) Facilitação da exploração e aprendizagem - avalia o grau em que a mãe incentiva comportamentos exploratórios do filho. A mãe com alto escore neste quesito estrutura o ambiente e as interações para promover a aprendizagem e o desenvolvimento de comportamentos exploratórios. A mãe com baixo escore neste domínio é ansiosa com a exploração do seu filho e não incentiva a exploração, independente do ambiente; (8) Conforto com contato físico - centra-se no quanto a mãe parece confortável em se engajar em contato físico com seu filho. Uma mãe com alto escore neste domínio mostra facilidade com a proximidade e contato e usa o contato corporal próximo durante momentos de angústia para acalmar o filho. A mãe com baixo escore parece estranha e desconfortável durante encontros íntimos com seu filho e usa, muitas vezes, objetos para mediar interações; (9) Envolvimento - avalia o grau em que a mãe se envolve ativamente com seu filho durante as interações. A mãe com alto escore neste domínio procura encontros sociais com a criança, provoca a atenção do filho e usa vocalizações ao longo de suas interações. A mãe com baixo escore não costuma iniciar interações e conversas com seu filho. Além dos escores em cada um dos domínios descrito acima, calculados a partir da média dos escores dos itens que compõe o domínio, outro escore gerado a partir do MBQS é o de sensibilidade materna. Ele é resultado da correlação entre os escores recebidos pela mãe durante a observação da interação com seu filho e os escores definidos por especialistas, referente a uma mãe fictícia considerada sensível. O coeficiente de correlação gerado, que varia de -1 a 1 , indica o quão próxima a mãe observada está de uma mãe considerada sensível, que percebe e interpreta as mensagens implícitas no comportamento da criança e responde a ela adequada e prontamente (Ainsworth, Blehar, Waters, \& Wall, 1978).

Durante a aplicação do MBQS, os 90 itens devem ser classificados através de um processo de escolha forçada e de maneira uniforme entre os nove escores possíveis de serem atribuídos ( 1 - extremamente não característico a 9 - extremamente característico). Ou seja, são escolhidos dez itens para cada escore do MBQS (para mais detalhes, consultar Becker, 2014).

Com relação aos vídeos, a análise foi realizada em duplas formadas pela primeira autora deste artigo, que analisou 
todos os casos, e também por três codificadores auxiliares, que analisaram um terço dos casos cada um. O treinamento dos codificadores foi conduzido pela primeira autora deste artigo e verificou-se uma porcentagem de concordância entre $70 \%$ e $90 \%$ (número de itens com escore igual ou com diferença de até dois pontos, dividido pelo número total de itens). Com a conclusão do treinamento, realizado com o uso de vídeos de outros casos do Projeto CRESCI, iniciou-se a análise dos vídeos de interação mãe-criança aos 12 e 18 meses do bebê. Cada dupla (codificador principal e auxiliar) assistiu ao vídeo de interação livre mãe-criança, realizou a codificação de forma independente e, em seguida, fez a comparação dos escores atribuídos. Verificou-se uma porcentagem de concordância entre os codificadores de 90,5\% e Índice de Correlação Intraclasse (ICC) de 0,88 nos vídeos dos 12 meses e também a porcentagem de concordância de 93,7\% e ICC de 0,92 nos vídeos dos 18 meses. Quando houve discordância entre os codificadores, foi necessário chegar a um acordo por meio de discussão acerca dos comportamentos observados.

\section{RESULTADOS}

Inicialmente, serão descritos os resultados referentes à interação mãe-criança nos Grupos Creche e Não Creche, com base nas observações realizadas nas fases dos $12 \mathrm{e}$ 18 meses e, em um segundo momento, apresentam-se os resultados sobre o desenvolvimento das crianças nos dois grupos, com base na avaliação realizada com as Escalas Bayley nas fases dos 6, 12 e 18 meses.

Um dos objetivos deste estudo foi investigar se haveria diferenças entre os grupos Grupos Creche e Não Creche na interação mãe-criança avaliados pelos nove domínios do MBQS (Pederson, Moran, \& Bento, 2013) nas fases dos 12 e 18 meses, bem como longitudinalmente entre as fases. Isto é, a interação mãe-criança seria diferente na fase dos 12 meses entre as crianças que há seis meses frequentavam a creche, quando comparadas ao outro grupo que não frequentava a creche? E depois de doze meses frequentando a creche, haveria alguma diferença entre os grupos? A hipótese inicial era de que o Grupo Creche, comparado ao Grupo Não Creche, apresentaria escores mais altos nos domínios de interação mãe-criança. Para tanto, foi realizada uma Análise Multivariada de Covariância (MANCOVA), usando a escolaridade materna como covariável, devido à correlação significativa dessa variável com alguns domínios de interação avaliados pela MBQS.

A MANCOVA revelou que a covariável teve efeito significativo na interação mãe-criança (Wilks's $\lambda=0,42$, $\mathrm{F}(10,32)=4,34, \mathrm{p}=0,001)$, mas que não houve diferenças significativas entre os grupos em nenhum dos nove domínios de interação examinados, seja na mesma fase (12 ou 18 meses) ou longitudinalmente entre elas. Tais resultados sugerem que eventuais diferenças nos escores de interação mãe-criança foram explicadas pela escolaridade materna. Ou seja, assim como as correlações indicaram, quanto maior a escolaridade da mãe, mais ela foi sensível às necessidades do filho ( $p<0,01$, fase dos 12 e 18 meses), apresentou eficácia de resposta ( $p<0,05$, em ambas as fases) e sincronia durante a interação ( $p<0,01$ em ambas as fases).

Os testes univariados, evidenciaram diferenças em dois domínios de interação, Eficácia de resposta e Facilitação da exploração e aprendizagem. Com relação ao domínio Eficácia de resposta, verificou-se o efeito de tempo - F(1, $41)=5,56, p<0,020 \mathrm{D}-$, indicando que em ambos os grupos houve aumento nos escores da fase dos 12 para a dos 18 meses. Como seria esperado, com o passar dos meses, as mães passaram a responder mais consistentemente e com eficácia aos sinais da criança, tanto os óbvios quanto os sutis. Quanto ao domínio Facilitação da exploração e aprendizagem, verificou-se efeito entre grupo e tempo, $\mathrm{F}(1,41)=11,67, p<0,001$, indicando que os escores do Grupo Creche aumentaram entre as fases dos 12 e dos 18 meses, enquanto os do Grupo Não Creche diminuíram neste período. Estes achados sugerem que as mães do Grupo Creche passaram a estruturar mais o ambiente e as interações, favorecendo a aprendizagem e o desenvolvimento de comportamentos exploratórios.

Outro objetivo do presente estudo foi investigar se haveria diferenças entre os grupos Creche e Não Creche nas Escalas Bayley III (Bayley, 2006) de desenvolvimento infantil em cada fase de coleta de dados (6, 12 e 18 meses), bem como longitudinalmente entre as fases. Isto é, o desenvolvimento infantil seria diferente na fase dos 12 meses entre as crianças que frequentavam há seis meses a creche, quando comparadas ao outro grupo que não frequentava a creche? E depois de doze meses frequentando a creche, haveria alguma diferença entre os grupos? A hipótese inicial era de que crianças que frequentaram a creche teriam escores mais elevados nas escalas de desenvolvimento cognitivo e da linguagem.

Para fins de análise, foi utilizado Análise Multivariada de Covariância 2x3 (MANCOVA), envolvendo dois grupos (Creche e Não Creche) e as três fases (6, 12 e 18 meses) como medida repetida. A idade da avaliação inicial, na fase dos 6 meses, foi utilizada como covariante, pois apresentou certa variação entre os dois grupos $(p<0,09)$ e correlação estatisticamente significativa com algumas escalas de desenvolvimento infantil.

A MANCOVA revelou que a covariável idade da avaliação inicial teve efeito significativo nos escores das escalas de desenvolvimento infantil (Wilks's $\lambda=0,68, F(4$, $37)=4,33, p=0,006)$. Isso indicou que, quanto mais idade tinha a criança na fase dos seis meses, maiores os seus escores nas escalas de Desenvolvimento cognitivo $(\mathrm{p}<0,01)$ e de Desenvolvimento da linguagem $(\mathrm{p}<0,05)$. Além disso, ocorreu um efeito de tempo entre as fases - Wilks's $\lambda=$ 
$0,34, \mathrm{~F}(8,33)=7,95, p<0,001)$. Por fim, apareceu também um efeito envolvendo a idade da avaliação inicial e as fases, (Wilks's $\lambda=0,36, \mathrm{~F}(8,33)=7,23, p<0,001)$. Para examinar em detalhes esses efeitos, foram analisados os testes univariados. A Tabela 2 apresenta detalhes dos resultados encontrados.

Verificou-se, assim, um efeito envolvendo as fases para a escala de Desenvolvimento cognitivo (efeito de tempo: $\mathrm{F}$ $(2,40)=15,4, p<0,01)$, indicando que houve diferença nos escores das crianças entre as fases. Para identificar as fases entre as quais houve diferença, realizou-se uma análise de covariância (ANCOVA), também considerando a idade da avaliação inicial como covariante. Contudo, essa análise não confirmou o efeito de tempo indicado na MANCOVA, uma vez que o teste post hoc (Bonferroni) não revelou diferença nos escore entre as fases. Outro efeito indicado pela análise multivariada foi o de idade da avaliação inicial e as fases de coleta $(\mathrm{F}(2,40)=15,2, p<0,01)$, revelando que, dependendo da idade da avaliação inicial das crianças, eram apresentados escores diferentes de Desenvolvimento cognitivo, ou seja, quanto mais meses a criança tinha na avaliação inicial, maior seu escore. Contudo, as médias dos escores de Desenvolvimento cognitivo não apresentaram diferença estatística em nenhuma das fases do estudo, não corroborando a hipótese que previa que a frequência à creche estaria associada a escores mais elevados de desenvolvimento cognitivo.

Os testes univariados também indicaram efeito envolvendo os grupos e as fases para a escala de Desenvolvimento cognitivo $(\mathrm{F}(2,40)=4,2, p=0,02)$, mas não se verificou diferença significativa nas médias desses grupos nas diferentes fases.

Quanto ao Desenvolvimento motor, foi observado efeito envolvendo as fases $(\mathrm{F}(2,40)=11,0, p<0,001)$, que revelou diferença nos escores das três fases investigadas. Para identificar as fases entre as quais houve diferença, realizou-se uma análise de covariância (ANCOVA), que revelou diferenças significativas nos escores de Desenvolvimento motor entre as fases dos 12 e 18 meses $(p=0,01)$, sendo que os escores na fase dos 18 meses foram mais elevados. Também se verificou efeito de grupo (F ( 2 , $40)=4,6, p=0,04)$, indicando que houve diferença entre os escores dos grupos Creche e Não Creche nos escores de Desenvolvimento motor. Para verificar em que fases as diferenças foram significativas, foram realizados Testes $t$ com correção de Bonferroni, para evitar a superestimação das diferenças. Foi constatado que houve diferença significativa nos escores dos grupos na fase dos 6 meses $(p$ $=0,006)$ e na fase dos 18 meses $(p=0,01)$, considerando que os escores do grupo Creche foram mais elevados do que o Não Creche nos dois momentos.

Por fim, na escala de Desenvolvimento motor também foi observado um efeito envolvendo a idade da avaliação inicial e as fases $(\mathrm{F}(2,40)=8,0, p<0,001)$, mostrando que, dependendo da idade da criança, elas apresentaram diferentes escores de desenvolvimento motor nas fases investigadas, embora não apareceram diferenças significativas nos escores de cada fase. Também foi encontrado um efeito envolvendo os grupos e as fases de coleta $(\mathrm{F}(2,40)=$ $5,9, p=0,006$ ), o qual indicou que os grupos Creche e Não Creche apresentaram diferentes escores ao longo do tempo. Os escores do Grupo Creche tenderam a decrescer entre as fases dos 6 e 12 meses e se elevar entre as fases dos 12 e 18 meses. Já nos escores do Grupo Não Creche, verificou-se uma elevação entre a fase dos 6 e 12 meses e um pequeno decréscimo aos 18 meses, confirmando o que já havia sido indicado anteriormente pelo Teste t, quando os escores do grupo Creche foram mais elevados do que os Não Creche.

Tabela 2

Escore médio, desvio padrão, valor de F e nível de significância para cada escala de desenvolvimento infantil da Bayley, entre os grupos (G) nas três fases (T), tendo como covariante a idade da avaliação inicial (I)

\begin{tabular}{|c|c|c|c|c|c|c|c|c|c|}
\hline & & \multicolumn{3}{|c|}{ Grupo Creche } & \multicolumn{3}{|c|}{ Grupo Não Creche } & \multirow{2}{*}{ F1 } & \multirow{2}{*}{$\mathbf{P}$} \\
\hline & & 6 meses & 12 meses & 18 meses & 6 meses & 12 meses & 18 meses & & \\
\hline \multirow[t]{2}{*}{ Cognitivo } & $\mathrm{M}$ & 103,6 & 101,2 & 98,3 & 98,7 & 102,6 & 101,3 & $\mathrm{~T}=15,37$ & 0,01 \\
\hline & DP & $(14,1)$ & $(6,9)$ & $(11,1)$ & $(14,3)$ & $(9,4)$ & $(11,3)$ & $\begin{array}{l}\mathrm{IxT}=15,19 \\
\mathrm{GxT}=4,16\end{array}$ & $\begin{array}{l}0,01 \\
0,02\end{array}$ \\
\hline \multirow[t]{2}{*}{ Linguagem } & $\mathrm{M}$ & 108,4 & 105,3 & 108,8 & 105,6 & 110,3 & 103,5 & \multirow{6}{*}{$\begin{array}{c}\mathrm{T}=11,03 \\
\mathrm{G}=04,75 \\
\mathrm{IxT}=7,99 \\
\mathrm{GxT}=5,93\end{array}$} & \multirow{2}{*}{$n s$} \\
\hline & DP & $(12,7)$ & $(10,8)$ & $(9,6)$ & $(11,0)$ & $(15,1)$ & $(16,3)$ & & \\
\hline \multirow[t]{2}{*}{ Motor } & M & 105,0 & 95,0 & 106,9 & 92,7 & 101,9 & 100,6 & & $\begin{array}{c}0,001 \\
0,03\end{array}$ \\
\hline & DP & $(16,0)$ & $(12,1)$ & $(7,7)$ & $(11,6)$ & $(16,6)$ & $(7,9)$ & & $\begin{array}{l}0,001 \\
0,006\end{array}$ \\
\hline \multirow[t]{2}{*}{ Socioemocional } & M & 104,8 & 105,0 & 105,5 & 104,3 & 107,9 & 106,4 & & \multirow{2}{*}{ is } \\
\hline & DP & $(11,7)$ & $(10,6)$ & $(11,6)$ & $(11,4)$ & $(10,6)$ & $(14,1)$ & & \\
\hline
\end{tabular}

Nota: Grupo Creche: $\mathrm{n}=21$; Grupo Não Creche: $\mathrm{n}=23 . \mathrm{G}=$ efeito principal para Grupo. $\mathrm{T}$ = efeito principal para Tempo (fase dos $12 \mathrm{e}$ fase dos 18 meses). GxT = efeito de interação Grupo x Tempo (fase dos 12 e fase dos 18 meses). IxT = efeito de interação Idade da avaliação inicial x Tempo (fase dos 12 e fase dos 18 meses). ${ }^{1} \mathrm{gl}=2,40$. 


\section{DISCUSSÃO}

Um dos objetivos do presente estudo foi investigar a existência de diferenças na qualidade da interação mãecriança entre díades em que as crianças frequentavam ou não a creche. A hipótese inicial foi de que o Grupo Creche apresentaria escores mais altos que o Grupo Não Creche, já que a literatura indica que poderia haver benefícios à interação, caso a creche apresentasse alta qualidade em seu atendimento (NICHD, 2003).

Todavia, tal hipótese não foi confirmada, uma vez que as análises estatísticas multivariadas, nas quais foi investigado o efeito conjunto da creche nos domínios de interação verificou-se a inexistência de diferenças significativas entre os grupos. Esses dados se aproximam do estudo de Booth et al. (2002), no qual as mães que cuidavam exclusivamente dos filhos, quando comparadas às mães que contavam com um cuidado alternativo, diferiram apenas no uso do tempo e não na qualidade da interação. Isso também parece ter ocorrido no presente estudo, pois apesar da qualidade da interação não mudar devido à frequência à creche, a forma que as mães utilizam o tempo com a criança pode ter sido diferente. As análises univariadas ainda indicaram que as mães do Grupo Creche parecem ter passado a priorizado mais as atividades de exploração e aprendizagem do que as mães do Grupo Não Creche.

Apesar de se perceber uma trajetória diferente entre os escores do Grupo Creche e Não Creche relacionados ao domínio Facilitação da Exploração, não foram verificadas diferenças significativas nas médias desses grupos. Assim, apenas se pode supor que as mães que tinham o filho na creche com o tempo apresentaram uma tendência a valorizar mais a exploração e aprendizagem do filho. Isto pode refletir a influência exercida pela creche, assim como explicitado no estudo do NICHD (2003), ao indicar que a mudança na qualidade da interação da mãe com seu filho pode ser influenciada pela interação dela com as educadoras na creche ou pode ser motivada pelas mudanças do filho em interação com as educadoras.

Portanto, cabe destacar que no presente estudo as horas que as crianças passaram longe de suas mães não foram suficientes para interferir na qualidade da interação entre as díades. Ou seja, a ida à creche parece não ter afetado a capacidade das mães em conhecer as especificidades do seu filho, suas necessidades e seu ritmo, um resultado que também foi encontrado por Booth et al. (2002). Por outro lado, o estudo do NICHD (2003) indicou que a qualidade da interação mãe-criança poderia ser favorecida se as crianças frequentassem creches de alta qualidade, pois a estreita relação entre pais e educadores incentivada nestas creches poderia favorecer a ambos em sua interação com a criança, além de as educadoras servirem de modelo de interação positiva. No caso do presente estudo, a qualidade das creches foi classificada como suficiente, o que pode não ter contribuído para impactar na qualidade da interação mãe-criança.

Outro fator que no presente estudo pode ter contribuído para as semelhanças encontradas entre os dois grupos diz respeito às características do Grupo Não Creche, que era composto por crianças que recebiam cuidados diversos em suas casas, mas não necessariamente cuidados exclusivos maternos. Sendo assim, muitas díades deste grupo também vivenciavam separações, por vezes, de várias horas diárias, aproximando os contextos de desenvolvimento dos dois grupos e minimizando as diferenças entre eles. Apesar de a creche não ter diferenciado a qualidade da interação mãe-criança entre os dois grupos, verificou-se diferença entre as fases dos 12 e 18 meses no domínio Eficácia de resposta, evidenciando que, em ambos os grupos, o escore avaliado na fase dos 18 meses foi significativamente maior que na fase dos 12 meses. Esse resultado pode indicar que, com o passar do tempo, as mães se tornaram mais eficientes em responder às necessidades dos filhos, seja porque elas passaram a conhecer mais os filhos e saber quais respostas eram mais eficazes para eles, seja porque a criança, com o passar do tempo, se tornou mais clara na manifestação de suas demandas, além de adquirir maior autocontrole, se satisfazendo mais facilmente com as respostas da mãe. Contudo, não foram verificadas diferenças nos demais domínios de interação, indicando que o padrão geral de interação mãe-criança tendeu a se manter estável, pelo menos ao longo do primeiro e segundo anos de vida.

Por fim, destaca-se que a análise multivariada indicou efeito da escolaridade da mãe sobre os domínios de interação mãe-criança, indicando que ela foi capaz de explicar possíveis diferenças na qualidade da interação entre as díades. Tal resultado corrobora outros estudos que indicam a influência da escolaridade materna na qualidade da interação mãe-criança (Hungerford \& Cox, 2006; NICHD, 2003; 2006b).

Com relação às diferenças no desenvolvimento infantil, a hipótese do estudo previa que, comparadas às crianças que não frequentaram a creche, crianças que frequentaram a creche teriam escores mais elevados de desenvolvimento infantil, em particular nas escalas de desenvolvimento cognitivo e da linguagem. Esta hipótese era baseada na literatura (NICHD, 2006a, 2006b; Ruzek et al., 2014; Sylva et al., 2011) e na ideia de que as experiências sociais e de aprendizagem propiciadas pela creche contribuiriam para o desenvolvimento infantil.

Os resultados do presente estudo não corroboraram esta hipótese. As análises revelaram que não houve diferenças significativas nos escores de desenvolvimento cognitivo e da linguagem da criança, com exceção do desenvolvimento motor, em que as crianças que ingressaram na creche apresentaram maior escore aos 18 meses. Apesar de a diferença encontrada indicar que a 
creche pode ter contribuído na diferenciação dos grupos quanto ao desenvolvimento motor da criança, essa área específica do desenvolvimento infantil não foi destacada nos estudos revisados. Isto sugere que as investigações em psicologia também poderiam incluir o desenvolvimento motor como variável importante a ser considerada tanto por si mesma como por potencializar as outras dimensões do desenvolvimento infantil.

Ao considerar que as hipóteses do estudo não foram corroboradas, destaca-se que uma possível explicação pode estar relacionada à qualidade das creches envolvidas no estudo. Como já indicado, essas creches apresentaram um nível suficiente de qualidade que, segundo Bairrão et al. (1997), indica a existência de condições mínimas de atendimento, porém, não sendo suficientes para a promoção de desenvolvimento infantil. Assim, constata-se que a qualidade das creches participantes se mostrou adequada para não causar prejuízos ao desenvolvimento infantil, mas insuficiente para oferecer benefícios e experiências expressivas para as crianças, a ponto de diferenciar os grupos Creche e Não Creche.

Outra possível explicação para as semelhanças encontradas entre os grupos pode estar relacionada ao fato de que o efeito da creche pode ter sido mediado pelo nível socioeconômico das famílias. A literatura indica que crianças provenientes de famílias com alta renda tendem a não se beneficiar da creche na mesma proporção que filhos de famílias com baixa renda (Geoffroy et al., 2007; Melhuish, 2004; Phillips \& Lowenstein, 2011). Por exemplo, o estudo de Loeb, Bridges, Bassok, Fuller e Rumberger (2007) indicou que crianças de famílias de classes média e alta que passavam mais de 30 horas semanais na creche não tiveram nenhum benefício para o seu desenvolvimento. Da mesma forma, as crianças participantes deste estudo também eram provenientes de famílias com renda média e alta e, entre elas, as que eram assíduas na creche passavam em torno de 40 horas semanais na instituição. Dessa forma, pode-se supor que famílias de classes média e alta são capazes de oferecer cuidados e estímulos necessários para o desenvolvimento infantil quando os filhos permanecem em casa, com capacidade de se igualar aos eventuais benefícios que a creche pode oferecer.

Para finalizar, cabe destacar algumas limitações relacionadas à amostra do estudo, cujas características representam uma parcela bastante específica da população, com mães, em geral, com elevado nível de escolaridade e com bom nível socioeconômico. É plausível supor que isso possa ter contribuído para amenizar o efeito da creche no desenvolvimento das crianças, somado ao fato de que a qualidade das creches envolvidas no presente estudo podem não ter sido suficiente para promover um desenvolvimento infantil diferenciado como se pode esperar em uma creche de alta qualidade (Burchinal et al., 2010; NICHD, 2002). É possível pensar que um nível suficiente de qualidade, como o deste estudo, poderia trazer benefícios para crianças provenientes de um contexto familiar com renda mais baixa, com mais limitações materiais, sociais e também emocionais (Geoffroy et al., 2007; Loeb et al., 2007; Melhuish, 2004; Oliveira et al., 2006; Phillips \& Lowenstein, 2011).

De modo geral, os resultados deste estudo indicaram que, para famílias que apresentam alta escolaridade e renda média ou alta, a creche não apresentou impacto expressivo, seja negativo ou positivo, tanto para a qualidade da interação mãe-criança como para o desenvolvimento infantil ao longo dos dois primeiros anos da criança. Sugere-se que futuros estudos avaliem o impacto de creches de alta qualidade em crianças provenientes de famílias de nível socioeconômico baixo.

\section{REFERÊNCIAS}

Ainsworth, M. D. S., Blehar, M., Waters, E., \& Wall, E. (1978). Patterns of attachment: A psychological study of the strange situation. Hillsdale, NJ: Erlbaum.

Albers, E. M., Riksen-Walraven, J. M., \& Weerth, C. (2010). Developmental stimulation in child care centers contributes to young infants' cognitive development. Infant Behavior \& Development, 33, 401-408.

Bairrão, J., Leal, T., Lima, I. A., \& Morgado, R. (1997). Educação pré-escolar. In Estudos temáticos, vol. $2-A$ evolução do sistema educativo e o PRODEP (pp. 21-110). Lisboa: Ministério da Educação.

Barros, R. P., Carvalho, M., Franco, S., Mendonça, R., \& Rosalém, A. (2011). Uma avaliação do impacto da qualidade da creche no desenvolvimento infantil. Pesquisa e Planejamento Econômico, 41(2), 213-232.

Bayley, N. (2006). Bayley Scales of Infant and Toddler Development - Third Edition, Administration Manual. San Antonio, TX: The Psychological Corporation.

Becker, S. M. S. (2014). Impacto da interação mãe-criança e da experiência de creche para o desenvolvimento infantil nos dois primeiros anos de vida da criança (Tese de doutorado não publicada). Curso de Pós-Graduação em Psicologia, Universidade Federal do Rio Grande do Sul, Porto Alegre, RS, Brasil.

Belsky, J. (2009). Classroom composition, child-care history and social development: Are child-care effects disappearing or spreading? Social Development, 18, 230-238.

Belsky, J., Vandell, D., Burchinal, M., Clarke-Stewart, K. A., McCartney, K., Owen, M., \& The NICHD Early Child Care Research Network. (2007). Are there long-term effects of early child care? Child Development, 78, 681-701.

Booth, C. L., Clarke-Stewart, K. A., Vandell, D. L., McCartney, K., \& Owen, M. T. (2002). Child-care usage and mother-infant "quality time". Journal of Marriage and Family, 64, 16-26.

Brasil. (1996). Lei de Diretrizes e Bases da Educação Nacional (LDB). Lei Federal n. ${ }^{\circ}$ 9.394, de 26/12/1996.

Burchinal, M., Vandergrift, N., Pianta, R., \& Mashburn, A. (2010). Threshold analysis of association between child care quality and child outcomes for low-income children in prekindergarten programs. Early Childhood Research Quarterly, 25(2), 166-176. 
Burger, K. (2010). How does early childhood care and education affect cognitive development? An international review of the effects of early interventions for children from different social backgrounds. Early Childhood Research Quarterly, $25,140-165$.

Camilli, G., Vargas, S., Ryan, S., \& Barnett, W. S. (2010). MetaAnalysis of the Effects of Early Education Interventions on Cognitive and Social Development. Teachers College Record, 112(3), 579-620.

Damiani, M. F., Dumith, S. D. C., Horta, B. L., \& Gigante, D. (2011). Educação infantil e longevidade escolar: dados de um estudo longitudinal. Estudos em Avaliação Educacional, 22(50), 515-533.

Geoffroy, M. C., Côté, S. M., Borge, A. I. H., Larouche, F., Séguin, J. R., \& Rutter, M. (2007). Association between nonmaternal care in the first year of life and children's receptive language skills prior to school entry: the moderating role of socioeconomic status. Journal of Child Psychology and Psychiatry, 48(5), 490-497.

Harms, T., Cryer, D. R., \& Clifford, R. M. (2003). Infant/Toddler Environment Rating Scale - Revised edition. New York: Teachers College Press.

Hungerford, A., \& Cox, M. J. (2006). Family factors in child care research. Evaluation Review, 30(5), 631-655.

Instituto Nacional de Estudos e Pesquisas Educacionais Anísio Teixeira. (2012). Censo da educação básica: 2011 - Resumo técnico. Brasília: Instituto Nacional de Estudos e Pesquisas Educacionais Anísio Teixeira.

Kude, V. M. M. (2004). Qualidade do atendimento na Educação Infantil: Um estudo em duas culturas. Educação, 53(2), 319-348.

Loeb, S., Bridges, M., Bassok, D., Fuller, B., \& Rumberger R. W. (2007). How much is too much? The influence of preschool centers on children's social and cognitive development. Economics of Education Review, 26, 52-66.

Lordelo, E. R. (1997). Efeitos da experiência de creche no desenvolvimento da criança: uma revisão. Psicologia: Teoria e Pesquisa, 13(2), 221-229.

Lordelo, E. R., Chalhub, A. A., Guirra, R. C., \& Carvalho, C. S. (2007). Contexto e desenvolvimento cognitivo: Frequência à creche e evolução do desenvolvimento mental. Psicologia: Reflexão e Crítica, 20(2), 324-334.

Melhuish, E. C. (2004). A literature review of the impact of early years provision upon young children, with emphasis given to children from disadvantaged backgrounds (Report to the Comptroller and Auditor General). London: National Audit Office.

Eunice Kennedy Shriver National Institute of Child Health and Human Development. (2002). Child-care structure $\rightarrow$ process $\rightarrow$ outcome: Direct and indirect effects of child-care quality on young children's development. Psychological Science, 13(3), 199-206.

Eunice Kennedy Shriver National Institute of Child Health and Human Development. (2003). Early child care and motherchild interaction from 36 months through first grade. Infant Behavior \& Development, 26, 345-370.

Eunice Kennedy Shriver National Institute of Child Health and Human Development. (2006a). Child-care effect sizes for the NICHD study of early child care and youth development. American Psychologist, 61(2), 99-116.

Eunice Kennedy Shriver National Institute of Child Health and Human Development. (2006b). Findings for children up to age $4 \frac{1}{2}$ years. Bethesda: Author.

Núcleo de Infância e Família/CRESCI. (2011a). Ficha de dados demográficos da família (Material não publicado). Porto Alegre, RS: Universidade Federal do Rio Grande do Sul.

Núcleo de Infância e Família/ CRESCI (2011b). Observação da Interação Mãe-Bebê - $6^{\circ}$ mês (Material não publicado). Porto Alegre, RS: Universidade Federal do Rio Grande do Sul.

Oliveira, E. A., Barros, F. C., Anselmi, L. D. S., \& Piccinini, C. A. (2006). The quality of home environment in Brazil: An ecological model. Journal of Child and Family Studies, 15(5), 633-644.

Pederson, D. R., Moran, G., \& Bento, S. (2013). The Maternal Behaviour Q-Sort (MBQS): Assessing maternal sensitivity and the quality of mother-infant interaction. London, Ontario: University of Western Ontario.

Phillips, D. A., \& Lowenstein, A. E. (2011). Early care, education, and child development. Annual Review of Psychology, 62, 483-500.

Piccinini, C. A., Lopes, R. R., Becker, S. M. S., Martins, G. D .F., Gabriel, M. R., Polli, R., \& Tudge, J. (2010). O impacto da creche no desenvolvimento socioemocional e cognitivo infantil: estudo longitudinal do sexto mês de vida do bebê ao final dos anos pré-escolares (Projeto de pesquisa não publicado). Porto Alegre, RS: Universidade Federal do Rio Grande do Sul.

Ruzek, E., Burchinal, M., Farkas, G., \& Duncan, G. J. (2014). The quality of toddler child care and cognitive skills at 24 months: Propensity score analysis results from the ECLS-B. Early Childhood Research Quarterly, 29, 12- 21.

Sylva, K., Stein, A., Leach, P., Barnes, J., Malmberg, L. E., \& The FCCC team. (2011). Effects of early child-care on cognition, language, and task-related behaviours at 18 months: An English study. British Journal of Developmental Psychology, 29(1), 18-45. 